\title{
Seeded Growth of Submicron Au Colloids with Quadrupole Plasmon Resonance Modes
}

Jessica Rodríguez-Fernández ${ }^{1}$, Jorge Pérez-Juste ${ }^{1, *}$, F. Javier García de Abajo ${ }^{2}$, and Luis M. Liz-Marzán ${ }^{1, *}$

\author{
${ }^{1}$ Departamento de Química Física, Universidade de Vigo, 36310 Vigo, Spain \\ Fax:+34 986812556. E-mail: juste@uvigo.es; lmarzan@uvigo.es \\ ${ }^{2}$ Centro Mixto CSIC-UPV/EHU and DIPC, Apartado 1072, 20080 San Sebastián, \\ Spain
}

\section{$\underline{\text { SUPPORTING INFORMATION }}$}

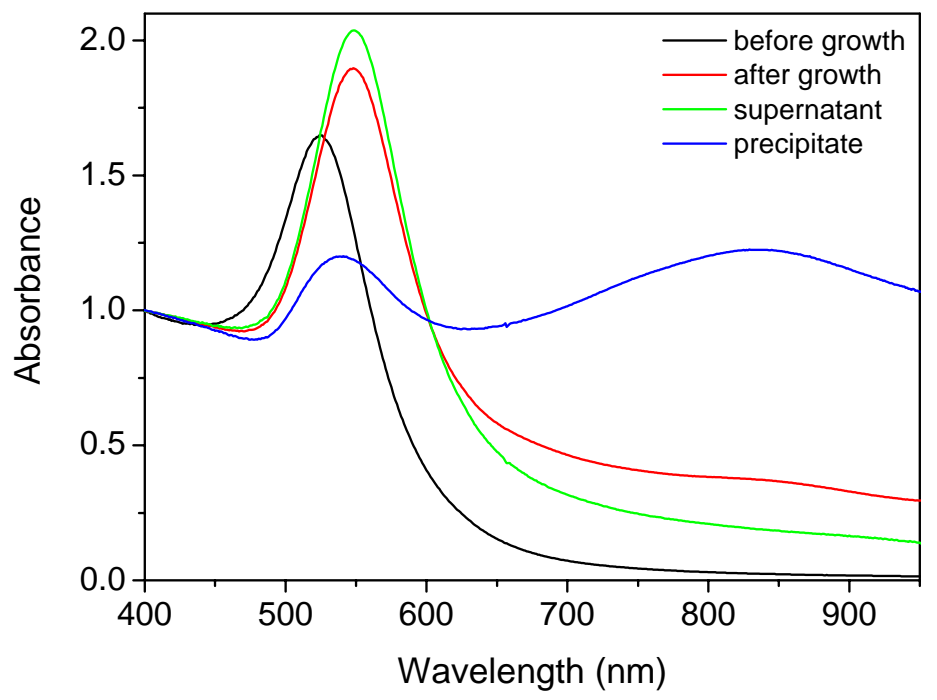

Figure S1. UV-vis spectra of the sample obtained after the first growth step, before (red) and after (green) purification. The blue spectrum was measured after redispersion of the precipitate. The spectrum of the seeds (black line) is shown as reference. All spectra were normalized at $400 \mathrm{~nm}$. 


\section{EXTERNAL FIELD}

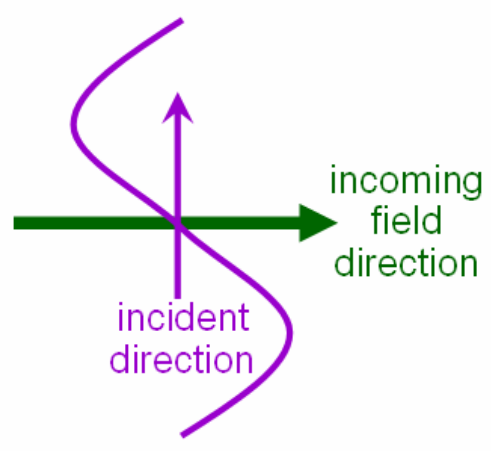

INDUCED CHARGES

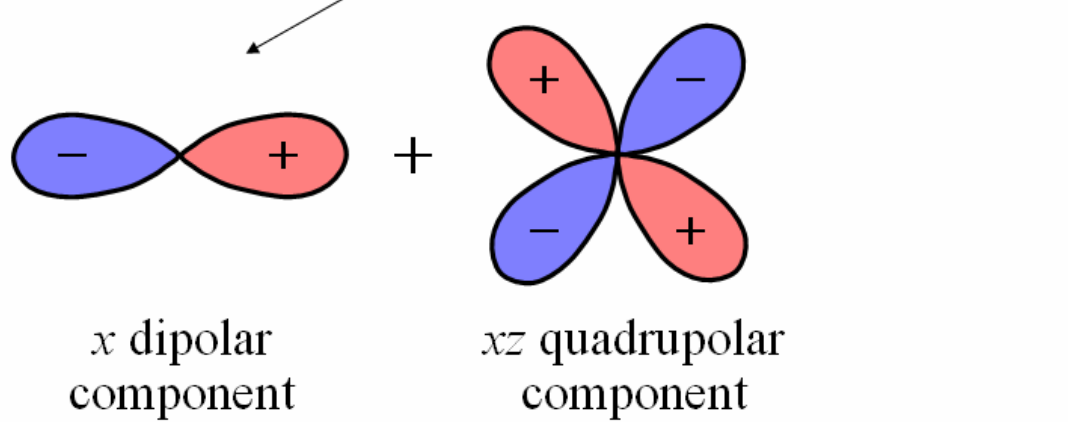

Figure S2. Illustration of the coupling of $x$-polarized incident light to $x$ and $x z$ multipolar components in the spheres. The scalar potential associated to the external field has both of these components, the $z$ dependence arising from the wave modulation along the propagation direction, which becomes noticeable as the particle size increases. In terms of multipoles, the $x$ component arises as a superposition of $(l=1, m= \pm 1)$, whereas the $x z$ component is a combination of $(l=2, m= \pm 1)$, and other values of the azimuthal number $m$ are forbidden by symmetry. 

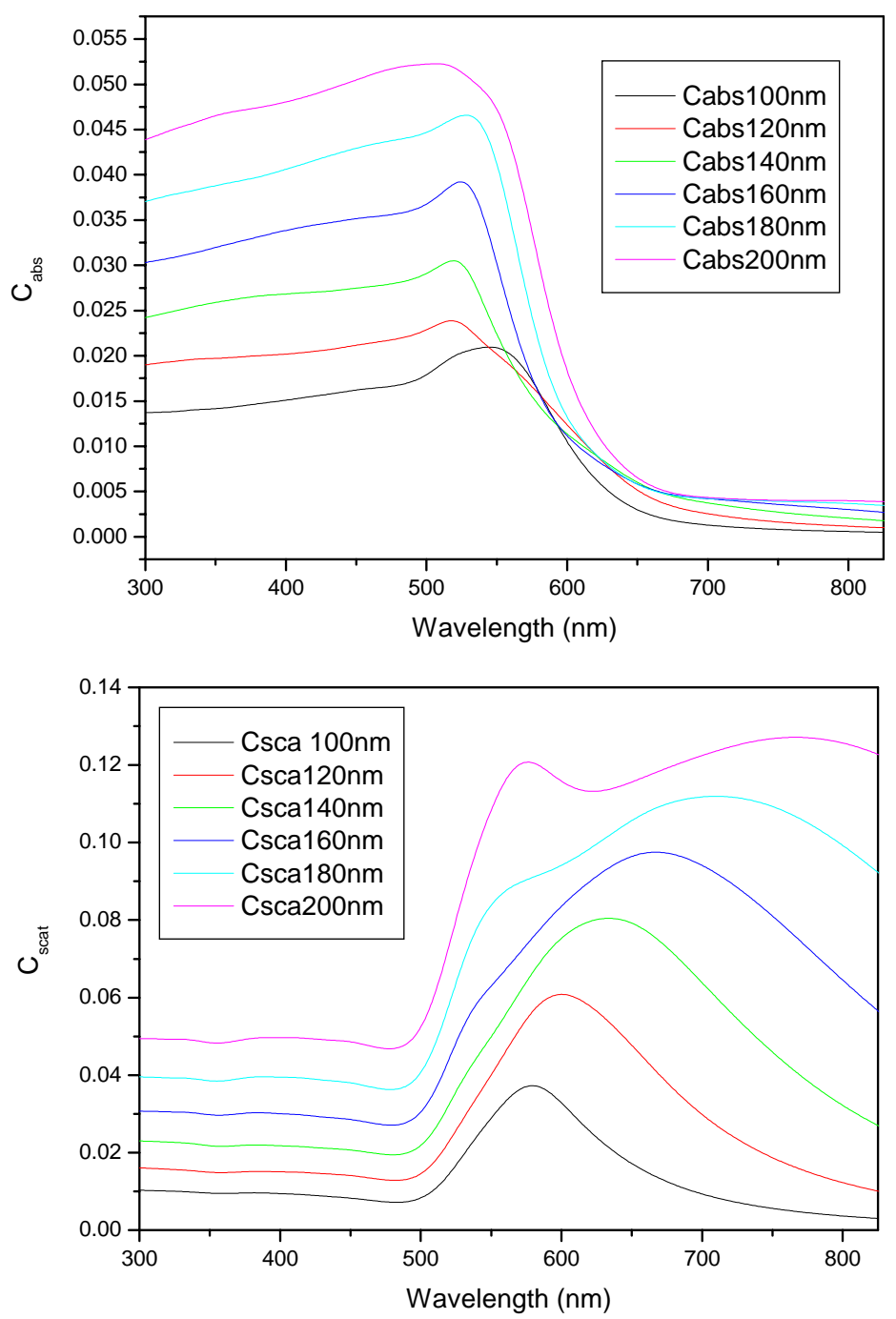

Figure S3. Calculated spectra for the absorption (top) and scattering (bottom) coefficients of gold nanospheres of different diameters, as indicated in the labels. 